Journal of Systems Science and Information

Oct., 2016, Vol. 4, No. 5, pp. 460-475

DOI: $10.21078 /$ JSSI-2016-460-16

\title{
Prescribed Performance Adaptive Control for a Class of Non-affine Uncertain Systems with State and Input Constraints
}

\author{
Longsheng CHEN \\ School of Aircraft Engineering, Nanchang Hangkong University, Nanchang 330063, China \\ E-mail: lschen2008@163.com \\ Qi WANG \\ School of Aircraft Engineering, Nanchang Hangkong University, Nanchang 330063, China \\ E-mail: wangqi439@126.com
}

\begin{abstract}
For a class of non-affine nonlinear systems with state constraints, input constraint, uncertain parameters and unknown external disturbance, a back-stepping control scheme is proposed based on mean value theorem, nonlinear mapping and prescribed performance bounds (PPB). The non-affine system is first transformed into a time-varying system with a linear structure by using the mean value theorem, and the intervals of the time-varying uncertain parameters are calculated. The bounded timevarying parameters and external disturbance are estimated by adaptive algorithms with projection; the estimation error is compensated by employing nonlinear damping technology. To handle the state and input constraints, the nonlinear mapping technique (NMT), hyperbolic tangent function and Nussbaum function are employed. The prescribed performance control method improves the performance of the system. It is proved that the proposed control scheme can guarantee that all signals of the closed-loop system are bounded through the Lyapunov analysis. Simulation results are presented to demonstrate the effectiveness of the proposed control scheme.
\end{abstract}

Keywords prescribed performance; state constraints; input constraints; non-affine system; nonlinear mapping; Nussbaum function

\section{Introduction}

Non-affine nonlinear systems widely exist in real world, such as biochemical process ${ }^{[1]}$, some aircraft dynamics $^{[2]}$, dynamic model in pendulum control ${ }^{[3]}$ and $\mathrm{AC} / \mathrm{DC}$ power system ${ }^{[4]}$, etc. In contrast to affine systems, non-affine systems do not have the control gains defined explicitly and the control inputs are embedded nonlinearly into the system through various possible ways. As such, control design for non-affine systems becomes an innovative and challenging topic ${ }^{[5,6]}$. Various attempts have been made in developing nonlinear control schemes with application to non-affine systems in the control area during the past decades. The hadamard transform ${ }^{[7]}$, mean value theorem ${ }^{[8,9]}$ and taylor series expansion ${ }^{[10-12]}$ have been used to transform the

Received November 27, 2015, accepted March 4, 2016

Supported by the Aeronautical Science Foundation of China (2015ZC560007), the Educational Commission of JiangXi Province of China (GJJ150707), the Natural Science Foundation of Jiangxi Province of China (20151BBE50026), National Natural Science Foundation of China (11462015) 
non-affine systems into affine form, then, combining with neural networks (NNs) and fuzzy logic systems (FLSs), the efficient nonlinear control schemes have been developed. However, some important nonlinear characteristics are lost in the process of affine transformation and the adaptability of controller is limit. In [13], a robust adaptive control scheme has been developed for a class of unknown structure, parameters and control direction non-affine system based on mean value theorem, adaptive algorithms with projection and nonlinear damping technique, where drop-out of nonlinear characteristics is avoided, but it is only effective just under the assumption that all the partial derivatives $\partial f(\cdot) / \partial x_{i}$ and $\partial f(\cdot) / \partial u$ are bounded on known constant closed intervals. Recently, directed control schemes which need not transform the non-affine system into affine form have been studied. Direct adaptive fuzzy or neural control schemes in [14-17] have been proposed for uncertain non-affine systems, where the implicit function theorem was exploited to assert the existence of continuous desired feedback controllers, and NNs or FLSs were used to approximate these desired feedback controllers. In [18-22], some direct control schemes have been presented by introducing a new unknown item which can make the input variables proportional to the so called "high frequency gain". Based on this, adaptive control schemes have been studied based on the combination of backstepping and NNs or FLSs, where NNs or FLSs are used to approximate the new unknown item $^{[18,19]}$. As we know, the approximation property of the NNs and FLSs is very complicated for the complex nonlinear system, and the new reconstruction error is introduced. In addition to these the approximation property of $\mathrm{NNs}$ is only effective just under the assumption that all input parameters of the NNs are inside the given bounded set, thus, not giving the proof of the system states boundedness, it is unreasonable to think that the up boundedness of the approximation errors exists. In [20,21], adaptive control schemes have been presented based on back-stepping, active disturbance rejection and extended state observer (ESO), where ESO was used to estimated the new unknown item. In [22], a low-cost and user-friendly PI-like control scheme is developed and a simple algorithm is adopted to estimate the new unknown item. However, the constraints of the input or states are ignored in aforementioned results. In real world, the constraints appear ubiquitously in the form of physical stoppages, saturation, safely specifications etc.

The existence of state and input constraints can degrade the system control performance, even leads to the system instability if they are ignored in the control design ${ }^{[23,24]}$. Furthermore, control design under consideration of state and input constraints is a challenging problem for any uncertain nonlinear system. Several schemes of control design for nonlinear systems with state and input constraints have been studied in recent years. Predictive control for nonlinear models (NMPC) has been applied to constraint systems successfully ${ }^{[25,26]}$. In [27], an antiwindup design is presented for single input adaptive control systems in strict feedback form with input saturation. In $[28,29]$, a robust adaptive control was proposed based on the back-stepping technique, using the special property of a hyperbolic tangent function and a Nussbaum function to deal with the input saturation. In [30,31], Neural networks were employed to approximate the input-output difference of actuators, so that a compensator was designed to overcome the input saturation. In recent years barrier Lyapunov function (BLF) has been introduced to handle constraints effectively ${ }^{[2,33]}$. By using BLF, the adaptive control was developed for 
nonlinear systems with full-state constraints ${ }^{[32]}$ and partial-state constraints ${ }^{[33]}$. However, the above mentioned control schemes are only designed for affine systems. In this paper, an adaptive control scheme for non-affine systems is developed to handle the input and state constraints based on hyperbolic tangent function and BLF. In order to overcome the problems caused by BLF, such as complex controller structure and extra parameters employment, the traditional BLF will be improved by nonlinear mapping technique (NMT).

On the other hand, the uncertainty and the state-input constraints will degrade the control performance, satisfactory control results must be guaranteed for the systems when they occur. In [34], an adaptive compensation control scheme for uncertain nonlinear systems with actuator failures was proposed based on prescribed performance bound transient performance. Prescribed performance adaptive control for MIMO affine nonlinear system was developed in [35]. In [36], Robust adaptive control was studied for feedback linearizable MIMO nonlinear systems with a prescribed performance. Robust decentralized adaptive stabilization was developed for interconnected systems with guaranteed transient performance in [37]. However, all the aforementioned results are obtained under the condition that the considered systems are affine nonlinear systems. In this study, a novel adaptive control is developed for non-affine systems with prescribed transient performance under the integrated influence of uncertainty and the state-input constraints.

Nowadays, for nonlinear systems, the back-stepping control is an effective technique, which utilizes the Lyapunov analysis to design the controller ${ }^{[28,38,39]}$. In [38-39], a robust and adaptive control scheme has been developed for a class of unknown nonlinear systems based on RBF neural network. However, with increasing relative order of the nonlinear system, the backstepping technique leads to the problems of "explosion of complexity". The differentiator ${ }^{[21,40]}$ has been proposed to solve the "explosion of complexity" in the back-stepping method, which avoids the need for computing the derivatives of virtual control laws and hence simplifies the design process.

Motivated by above discussion and analysis, the adaptive tracking control scheme is developed for non-affine system in the presence of parameter uncertainty, state constraints, input constraint and unknown external disturbance based on back-stepping technique. Compared with the existing literature, the following contributions are worth to be emphasized.

(i) The studied plant is a class of non-affine systems in the presence of state and input constraints, there are very few works for solving multi-constraints of non-affine system at present.

(ii) On the premise of no drop-out of nonlinear characteristics, the non-affine system is transformed into a time-varying system with a linear structure by employing the mean value theorem with further attenuated assumptions, and all the constraints are taken into account.

(iii) Based on improved BLF which is called NMT in this paper, requirements on states is satisfied by mapping the constrained states into the real number set; then, the controller is designed by combining hyperbolic tangent function with Nussbaum function, the designed algorithm can not only deal with the input constraint but also the possible "controller singularity" problem causing by unknown control direction.

(iv) The proposed scheme is robust, adaptable, fault-tolerant and universal without the need for any approximation to the non-affine system in deriving the control strategy. The design 
procedure is not complicated, which reduces the computation load and makes it easy in actual implementation.

The organization of the paper is as follows. Section 2 describes the problem and Section 3 presents the design procedure about NMT and PPB. The adaptive tracking control scheme is investigated for non-affine system by considering parameter uncertainty, state constraints, input constraint and unknown external disturbance in Section 4. The simulation example is given to illustrate the effectiveness of the proposed scheme in Section 5, and followed by Section 6 which concludes the work.

\section{Problem Statement}

Consider a class of uncertain nonaffine nonlinear systems with state and input constraints which is described by

$$
\left\{\begin{array}{l}
\dot{x}_{i}=x_{i+1}, \quad 1 \leq i \leq n-1, \\
\dot{x}_{n}=f(\boldsymbol{x}, u(\nu))+d_{m}(t), \\
y=x_{1},
\end{array}\right.
$$

where $\boldsymbol{x}=\left[x_{1}, x_{2}, \cdots, x_{n}\right]^{\mathrm{T}} \in R^{n}, \nu \in R$ and $y \in R$ are the system states, input and output, respectively. $d_{m}(t)$ is the unknown external disturbance. $f(\cdot)$ is a smooth nonlinear function with known structure and parameter uncertainty. We consider system (1) with state constraints, i.e., $\forall t \geq 0, x_{i}(i=1,2, \cdots, n)$ is required to remain in the set $\left|x_{i}\right| \leq \ell_{i}$, where $\ell_{i}$ is a positive constant. The input constraint $u(\nu)$ can be express as the follow saturated function

$$
u(\nu)=\operatorname{sat}(\nu)= \begin{cases}\operatorname{sign}(\nu) u_{M}, & |\nu| \geq u_{M}, \\ \nu, & |\nu|<u_{M},\end{cases}
$$

where $u_{M}$ is the bound of $u(\nu)$. In this paper, the control objective is that the adaptive tracking control scheme is developed to retain the system output $y$ to follow the given desired trajectory $y_{r}$ in the presence of parameter uncertainty, state constraints, input constraint and unknown external disturbance. To facilitate the control system design, the following assumptions, definitions and lemmas are required.

Assumption 1 Nonlinear function $f(\cdot)$ is continuously differentiable and the system states are all available for feedback.

Assumption 2 The system uncertain parameters are bounded by known positive constants or the uncertain parameters influence system performance by bounded functions, such as sinusoidal function, cosine function, and so on.

Assumption 3 The disturbance $d_{m}(t)$ is bounded by a positive constant $d_{m}$, i.e., $\left|d_{m}(t)\right| \leq$ $d_{m}$.

Assumption $4 \forall t \geq 0$, the partial derivative $\partial f(\cdot) / \partial u \neq 0$, and the initial value of $f(\cdot)$ is bounded by a positive constant $d_{0}$, i.e., $|f(0,0, \cdots, 0)| \leq d_{0}$.

Assumption 5 For the uncertain nonaffine system (1), the bound $u_{M}$ and $\ell_{i}$ are known.

Assumption 6 There exists a positive constant $\ell_{r}$ satisfying $\ell_{r}<\ell_{1}$ such that reference signal $y_{r}$ is bounded by $\ell_{r}$, i.e., $\left|y_{r}\right| \leq \ell_{r}$. 
Definition 1 (see [41]) If the continuous function $N(\kappa): R \rightarrow R$ satisfying the following conditions: (i) $\lim _{s \rightarrow \infty} \sup \frac{1}{s} \int_{0}^{s} N(\kappa) \mathrm{d} \kappa=+\infty$; and (ii) $\lim _{s \rightarrow \infty} \inf \frac{1}{s} \int_{0}^{s} N(\kappa) \mathrm{d} \kappa=-\infty$. Then $N(\kappa)$ is called a Nussbaum function.

Lemma 1 (see [13]) Let $V(\cdot)$ and $\kappa(\cdot)$ be smooth functions defined on $\left[0, t_{f}\right)$ with $V(t) \geq 0$, $\forall t \in\left[0, t_{f}\right)$, and $N(\cdot)$ is a smooth Nussbaum-type function. If the following inequality holds

$$
0 \leq V(t) \leq \delta_{0} \pm \mathrm{e}^{-\delta t} \int_{0}^{t}(g(x(\tau)) N(\kappa)+1) \dot{\kappa} \mathrm{e}^{\delta t} \mathrm{~d} \tau, \quad \forall t \in\left[0, t_{f}\right),
$$

where $\delta_{0}$ represents some suitable constant, $\delta$ is a positive constant, and $g(x(\tau)$ is a time-varying parameter which takes values in the unknown closed intervals $\Re:=\left[I^{-}, I^{+}\right]$, with $0 \notin \Re$, then $V(t), \kappa(t)$ and $\int_{0}^{t}(g(x(\tau)) N(\kappa)+1) \dot{\kappa} \mathrm{d} \tau$ must be bounded on $\left[0, t_{f}\right)$.

Lemma 2 (see [13]) Suppose $0 \leq t_{f} \leq \infty$ and that $x:\left[0, t_{f}\right] \rightarrow R^{N}$ is a solution of the closed loop system. If $x$ is a bounded solution, then $t_{f}=\infty$.

Since $f(\cdot)$ is continuously differentiable and the $f(0, \cdots, 0,0)$ is bounded, the $f(\cdot)$ can be rewritten as the following form by using mean value theorem.

$$
\begin{aligned}
f(\boldsymbol{x}, u)= & f(\boldsymbol{x}, u)-f\left(0, x_{2}, \cdots x_{n}, u(\nu)\right)+f\left(0, x_{2}, \cdots x_{n}, u(\nu)\right) \\
& -f\left(0,0, x_{3}, \cdots x_{n}, u(\nu)\right)+\cdots+f(0, \cdots 0, u(\nu))-f(\mathbf{0})+f(\mathbf{0}) \\
= & b_{1}(t) x_{1}+\cdots+b_{n}(t) x_{n}+\beta(t) u(\nu)+f(\mathbf{0})+d_{m}(t),
\end{aligned}
$$

where $f(\mathbf{0})$ is the abbreviation for $f(0,0, \cdots, 0)$, and

$$
b_{i}(t)=\left.\frac{\partial f}{\partial x_{i}}\right|_{\left(0, \cdots, 0, \gamma_{i}, x_{i+1}, \cdots, x_{n}, u(v)\right)}, \quad i=1,2, \cdots, n, \quad \beta(t)=\left.\frac{\partial f}{\partial u}\right|_{\left(0, \cdots, 0, \gamma_{u}\right)}, \quad \beta(t) \neq 0,
$$

where $\gamma_{i} \in\left[0, x_{i}\right](i=1,2, \cdots, n)$, and $\gamma_{u} \in[0, u(\nu)]$. In this paper, it does not concern the $\gamma_{i}$, and $\gamma_{u}$ and the concrete form of $b_{i}(t), \beta_{i}(t)$. In the procedure of control design, it only needs the constraint intervals of $b_{i}(t), \beta_{i}(t)$. Combining the mean value theorem with state and input constraints, the obtained method of constraint intervals for $b_{i}(t), \beta_{i}(t)$ is shown as follows.

(i) According to the characteristics of the mean value theorem, it is obvious that the time varying parameters $b_{i}(t)$ and $\beta_{i}(t)$ are the nonlinear function of the state $\boldsymbol{x}$. input $u$ and uncertain parameters.

(ii) According to Assumptions 2 and 5, the system input, states and uncertain parameters are constrained by known constants, So the problem of solving the constraint intervals for $b_{i}(t)$ and $\beta_{i}(t)$ is essentially extreme-value problem of multi-variable functions. It is easy to gain the extreme-values by the Lagrange method, i.e., $b_{i}(t) \in\left[b_{i \min }, b_{i \max }\right]$ and $\beta(t) \in\left[\beta_{\min }, \beta_{\max }\right]$.

(iii) The detailed solution procedure of the constraint intervals for $b_{i}(t)$ and $\beta_{i}(t)$ will be given in the simulation. The constraint intervals of $b_{i}(t)$ and $\beta_{i}(t)$ are based on the constraint conditions $\left|x_{i}\right|<\ell_{i}$ and $|u(\nu)| \leq u_{M}$, so the developed scheme must satisfy these constraints.

Considering that the saturation is approximated by a hyperbolic tangent function, the $\operatorname{sat}(\nu)$ in (2) can be expressed as

$$
\begin{aligned}
& \operatorname{sat}(v)=h(v)+d_{v}(t), \\
& h(v)=u_{M} \times \tanh \left(\frac{\nu}{u_{M}}\right)=u_{M} \frac{\mathrm{e}^{\nu / u_{M}}-\mathrm{e}^{-\nu / u_{M}}}{\mathrm{e}^{\nu / u_{M}}+\mathrm{e}^{-\nu / u_{M}}},
\end{aligned}
$$


where $d_{v}(t)=\operatorname{sat}(v)-h(v)$ is a bounded function and the bound of $d_{v}(t)$ satisfies

$$
\left|d_{v}(t)\right| \leq u_{M}(1-\tanh (1)) .
$$

Considering (4)-(7), the uncertain nonaffine system(1) can be rewritten as

$$
\left\{\begin{array}{l}
\dot{x}_{i}=x_{i+1}, \quad 1 \leq i \leq n-1 \\
\dot{x}_{n}=b_{1}(t) x_{1}+\cdots+b_{n}(t) x_{n}+\beta(t) h(\nu)+f(\mathbf{0})+d(t), \\
y=x_{1}
\end{array}\right.
$$

where $d(t)=d_{m}(t)+\beta d_{v}(t)$. It is obvious that the $d(t)$ is also bounded by a positive constant $d$, i.e., $|d(t)| \leq d$ and $d=\max (|\beta|) u_{M}(1-\tanh (1))+d_{m}$.

\section{Nonlinear Mapping Technique and Prescribed Performance Bounds}

\subsection{Nonlinear Mapping Technique (NMT)}

If the control scheme is developed based on system (8), it may not satisfy the requirements on states. So the NMT is employed to map the constrained states into the real number set.

Defining one to one mapping $H: x_{i} \rightarrow x_{i}^{*}$ as follow

$$
x_{i}^{*}=\ln \frac{\ell_{i}+x_{i}}{\ell_{i}-x_{i}}
$$

where $H$ is elementary function. According to (9), we obtain

$$
x_{i}=\mathrm{H}^{-1}=\ell_{i} \frac{\mathrm{e}^{x_{i}^{*}}-1}{\mathrm{e}^{x_{i}^{*}}+1} .
$$

Differentiating $x_{i}$ with respect to time yields

$$
\dot{x}_{i}=\frac{2 \ell_{i} \mathrm{e}^{x_{i}^{*}} \dot{x}_{i}^{*}}{\left(\mathrm{e}^{x_{i}^{*}}+1\right)^{2}} .
$$

Defining $g\left(x_{i}^{*}\right)=\left(\mathrm{e}^{x_{i}^{*}}+\mathrm{e}^{-x_{i}^{*}}+2\right)(i=1,2, \cdots, n)$ and $h\left(x_{i+1}^{*}\right)=\left(\mathrm{e}^{x_{i+1}^{*}}+1\right)^{-1}(i=1,2, \cdots, n-1)$, it is obvious that $g\left(x_{i}^{*}\right)>0$ and $h\left(x_{i+1}^{*}\right)>0$, substituting (10) and (11) into (8), the system (8) can be rewritten as the following form without any constraint:

$$
\left\{\begin{array}{l}
\dot{x}_{i}^{*}=\frac{\ell_{i+1}}{2 \ell_{i}} g\left(x_{i}^{*}\right)-\frac{\ell_{i+1}}{\ell_{i}} g\left(x_{i}^{*}\right) h\left(x_{i+1}^{*}\right), \quad i=1,2, \cdots, n-1, \\
\dot{x}_{n}^{*}=\sum_{i}^{n} \frac{b_{i} \ell_{i} g\left(x_{n}^{*}\right)}{2 \ell_{n}} \frac{\mathrm{e}^{x_{i}^{*}}-1}{\mathrm{e}^{x_{i}^{*}}+1}+\frac{g\left(x_{n}^{*}\right)}{2 \ell_{n}}(\beta u(\nu)+f(\mathbf{0})+d(t)) .
\end{array}\right.
$$

According to Assumption 6, the reference signal also be bounded by the $\ell_{1}$, so we define

$$
y_{r}^{*}=\log \frac{\ell_{1}+y_{r}}{\ell_{1}-y_{r}} .
$$

Thus, system (1) with state and input constraints can be equated to system (12) without any constraint, and the reference signal is $y_{r}^{*}$ in (13). Now, we can use backstepping technique directly to design control scheme based on (12) and (13). Firstly, we introduce the following lemma.

Lemma 3 For the uncertain nonlinear systems (1) and (12), $P=\left\{x_{i}(t):-\ell_{i}<x_{i}(t)<\right.$ $\left.\ell_{i}\right\}, Q=\left\{x_{i}^{*}(t): x_{i}^{*}(t) \in R\right\}$ and mapping $H: x_{i} \rightarrow x_{i}^{*}$, if $x_{i}^{*} \rightarrow q_{i}(t)$, the $x_{i}(t)$ satisfies $x_{i}(t) \rightarrow p_{i}(t)=H^{-1}\left(q_{i}(t)\right)$. 
Proof Because $H$ is a continuous elementary function in its domain, the inverse mapping $\mathrm{H}^{-1}$ is also a continuous elementary function. Defining $e_{i}^{*}=x_{i}^{*}-q_{i}(t)$ and $e_{i}=x_{i}-p_{i}(t)$, invoking (9) and (10), then $e_{i}$ can be expressed as

$$
e_{i}=x_{i}-p_{i}(t)=\ell_{i} \frac{\mathrm{e}^{x_{i}^{*}}-1}{\mathrm{e}^{x_{i}^{*}}+1}-\ell_{i} \frac{\mathrm{e}^{q_{i}(t)}-1}{\mathrm{e}^{q_{i}(t)}+1}=\frac{2 \ell_{i}\left(\mathrm{e}^{x_{i}^{*}}-\mathrm{e}^{q_{i}(t)}\right)}{\left(\mathrm{e}^{x_{i}^{*}}+1\right)\left(\mathrm{e}^{q_{i}(t)}+1\right)}
$$

then

$$
\lim _{e_{i}^{*} \rightarrow 0} e_{i}=\lim _{x_{i}^{*} \rightarrow q_{i}(t)} \frac{2 \ell_{i}\left(\mathrm{e}^{x_{i}^{*}}-\mathrm{e}^{q_{i}(t)}\right)}{\left(\mathrm{e}^{x_{i}^{*}}+1\right)\left(\mathrm{e}^{q_{i}(t)}+1\right)}=0 .
$$

So if $x_{i}^{*} \rightarrow q_{i}(t)$, the $x_{i}(t) \rightarrow p_{i}(t)=H^{-1}\left(q_{i}(t)\right)$.

Remark 1 In [32] and [33], the BLF is incorporated into Lyapunov to handle the problem of state constraints. In this paper, the nonlinear mapping technique will be adopted to overcome the problems faced by BLF, such as complex controller structure, small set for initial value and extra parameters employment.

\subsection{Prescribed Performance Bounds (PPB)}

The objective in this section is to ensure the transient performance in the sense that the tracking error $e(t)=y(t)-y_{r}(t)$ is preserved within a specified PPB all the time. In [33-37], PPB transient performance was guaranteed irrespective of the presence of nonaffine characteristic and state constraints. Similar to the results developed in [33-37], the characterization of a prescribed performance bound is required. In this situation, let the tracking error satisfying the condition that

$$
-\underline{\varsigma} \rho(t)<z(t)<\bar{\varsigma} \rho(t), \quad \forall t \geq 0,
$$

where $z(t)=y^{*}(t)-y_{r}^{*}(t)$ and $0<\underline{\varsigma}, \bar{\varsigma} \leq 1 . \quad \rho(t)$ is performance to be designed, and $\rho(t)$ is a decreasing function with $\rho_{0}>\lim _{t \rightarrow \infty} \rho(t)=\rho_{\infty}>0$. For example, $\rho(t)$ can be chosen as $\rho(t)=\left(\rho_{0}-\rho_{\infty}\right) \mathrm{e}^{-l t}+\rho_{\infty}$, where $l>0$. Considering the nonlinear mapping, the $\rho_{0}$ is designed as $\rho_{0} \geq|z(0)|$ in this study.

Considering the constrained condition (16) in the tracking error, it can be transformed into solving a problem with the boundedness of the signals as the only requirement. The essential task is transformation the original nonlinear system with the constrained (16) tracking error behavior into an equivalent unconstrained one. To complete these, a smooth and strictly increasing function $T(\varepsilon)$ is introduced, which has the following properties ${ }^{[34]}$ :

(i) $-\underline{\varsigma}<T(\varepsilon)<\bar{\varsigma}$; (ii) $\lim _{\varepsilon \rightarrow+\infty} T(\varepsilon)=\bar{\varsigma}, \quad \lim _{\varepsilon \rightarrow-\infty} T(\varepsilon)=\underline{\varsigma}$; (iii) $T(0)=0$.

In accordance with properties (i) and (ii) of the transformed function $T(\varepsilon)$, the performance condition (16) can be expressed as

$$
z(t)=\rho(t) T(\varepsilon)
$$

In this study, the transformed function $T(\varepsilon)$ is designed as

$$
T(\varepsilon)=\frac{\bar{\varsigma} \mathrm{e}^{(\varepsilon+r)}-\underline{\varsigma} \mathrm{e}^{-(\varepsilon+r)}}{\mathrm{e}^{(\varepsilon+r)}+\mathrm{e}^{-(\varepsilon+r)}}
$$

where $r=\ln (\underline{\varsigma} / \bar{\varsigma}) / 2$. It is obvious that the $T(\varepsilon)$ has the properties (i)-(iii). From (17) and (18), the transformed error $\varepsilon$ is solved as

$$
\varepsilon=0.5 \ln (\bar{\varsigma} \gamma(t)+\underline{\varsigma} \bar{\varsigma})-0.5 \ln (\underline{\varsigma} \bar{\varsigma}-\underline{\varsigma} \gamma(t))
$$


where $\gamma(t)=z(t) / \rho(t)$. Considering (19), the time derivative of $\varepsilon$ is

$$
\dot{\varepsilon}=\frac{\partial T^{-1}}{\partial \gamma} \dot{\gamma}=0.5\left[\frac{1}{\gamma+\underline{\varsigma}}-\frac{1}{\gamma-\bar{\varsigma}}\right]\left(\frac{\dot{z}}{\rho}-\frac{z \dot{\rho}}{\rho^{2}}\right)=\xi\left(\dot{y}^{*}-\dot{y}_{r}^{*}-\frac{z \dot{\rho}}{\rho}\right),
$$

where $\xi$ is defined as

$$
\xi=\frac{1}{2 \rho}\left[\frac{1}{\gamma+\underline{\varsigma}}-\frac{1}{\gamma-\bar{\varsigma}}\right]
$$

The PPB can now be incorporated into the system (1), considering the NMT, Equations (12), (13) and (20), the system (1) can be rewritten as the following form by replacing the equation for $\dot{x}_{1}^{*}$ with $\dot{\varepsilon}$.

$$
\left\{\begin{array}{l}
\dot{\varepsilon}=\xi\left(\frac{\ell_{2}}{2 \ell_{1}} g\left(x_{1}^{*}\right)-\frac{\ell_{2}}{\ell_{1}} g\left(x_{1}^{*}\right) h\left(x_{2}^{*}\right)-\dot{y}_{r}^{*}-\rho^{-1} \dot{\rho} z\right), \\
\dot{x}_{i}^{*}=\frac{\ell_{i+1}}{2 \ell_{i}} g\left(x_{i}^{*}\right)-\frac{\ell_{i+1}}{\ell_{i}} g\left(x_{i}^{*}\right) h\left(x_{i+1}^{*}\right), \quad i=2,3, \cdots, n-1, \\
\dot{x}_{n}^{*}=\sum_{i}^{n} \frac{b_{i} \ell_{i} g\left(x_{n}^{*}\right)}{2 \ell_{n}} \frac{\mathrm{e}^{x_{i}^{*}}-1}{\mathrm{e}^{x_{i}^{*}}+1}+\frac{g\left(x_{n}^{*}\right)}{2 \ell_{n}}(\beta u(\nu)+f(\mathbf{0})+d(t)) .
\end{array}\right.
$$

\section{Adaptive Tracking Control Design Based on Backstepping}

In this section, the adaptive tracking control scheme will be proposed based on adaptive algorithms with projection, nonlinear damping technique and differentiator by employing backstepping technique for the uncertain nonaffine system (1), according the aforementioned technique, such as the mean value theorem, hyperbolic tangent function, nonlinear mapping and PPB, the control design for system (1) is equated to control design for system(20). The detail design process is described as follows.

Step 1 To develop the backstepping control with guaranteed transient performance, the following variables are defined.

$$
z_{1}=\varepsilon, \quad z_{2}=\alpha_{1}-h\left(x_{2}^{*}\right),
$$

where $\alpha_{1}$ is a designed virtual control law. Considering the first equation of (22), the second equation of (23) and differentiating $z_{1}$ yields

$$
\dot{z}_{1}=\dot{\varepsilon}=\xi\left(\frac{\ell_{2}}{2 \ell_{1}} g\left(x_{1}^{*}\right)-\frac{\ell_{2}}{\ell_{1}} g\left(x_{1}^{*}\right)\left(\alpha_{1}-z_{2}\right)-\dot{y}_{r}^{*}-\rho^{-1} \dot{\rho} z\right) .
$$

The virtual control law is designed as

$$
\alpha_{1}=-\frac{\ell_{1}}{\ell_{2} g\left(x_{1}^{*}\right)}\left(-c_{1} \xi^{-1} z_{1}+\dot{y}_{r}^{*}+\rho^{-1} \dot{\rho} z-\frac{\ell_{2} g\left(x_{1}^{*}\right)}{2 \ell_{1}}\right),
$$

where $c_{1}>0$ is a designed constant. Substituting (25) into (24) yields

$$
\dot{z}_{1}=-c_{1} z_{1}+\frac{\xi \ell_{2}}{\ell_{1}} g\left(x_{1}^{*}\right) z_{2} .
$$

Consider the Lyapunov function as

$$
V_{1}=0.5 z_{1}^{2} .
$$

Considering (27), the time derivative of $V_{1}$ is given by

$$
\dot{V}_{1}=-c_{1} z_{1}^{2}+\frac{\xi \ell_{2}}{\ell_{1}} g\left(x_{1}^{*}\right) z_{1} z_{2} .
$$


It is apparent that the first term on the right-hand side is stable in (28). Furthermore, the second term will be canceled in the next step.

Remark 2 In this paper, the $h\left(x_{i+1}^{*}\right)$ is deemed as virtual controlled variable, and it is different from the traditional back-stepping in which $x_{i+1}^{*}$ is deemed as virtual controlled variable.

Step 2 Considering second subsystem of (22) and differentiating $z_{2}$ in (23) with respect to time yields

$$
\dot{z}_{2}=\dot{\alpha}_{1}-\frac{\mathrm{d} h\left(x_{2}^{*}\right)}{\mathrm{d} t}=\dot{\alpha}_{1}+\frac{\ell_{3}}{2 \ell_{2}}-\frac{\ell_{3}}{\ell_{2}} h\left(x_{3}^{*}\right)
$$

The virtual control law is designed as

$$
\alpha_{2}=-\frac{\ell_{2}}{\ell_{3}}\left(-c_{2} z_{2}-\dot{\alpha}_{1}-\frac{\xi \ell_{2}}{\ell_{1}} g\left(x_{1}^{*}\right) z_{1}-\frac{\ell_{3}}{2 \ell_{2}}\right),
$$

where $c_{2}>0$ is designed constants. Defining

$$
z_{3}=\alpha_{2}-h\left(x_{3}^{*}\right)
$$

Substituting (30) and (31) into (29) yields

$$
\dot{z}_{2}=-c_{2} z_{2}+\frac{\ell_{3}}{\ell_{2}} z_{3}-\frac{\xi \ell_{2}}{\ell_{1}} g\left(x_{1}^{*}\right) z_{1}
$$

Consider the Lyapunov function as

$$
V_{2}=0.5 z_{2}^{2}
$$

Considering (32), the time derivative of $V_{2}$ is given by

$$
\dot{V}_{2}=-c_{2} z_{2}^{2}+\frac{\ell_{3}}{\ell_{2}} z_{2} z_{3}-\frac{\xi \ell_{2}}{\ell_{1}} g\left(x_{1}^{*}\right) z_{1} z_{2} .
$$

Step $i(i=3,4, \cdots, n-1) \quad$ Similar to Step 2, Defining

$$
z_{i}=\alpha_{i-1}-h\left(x_{i}^{*}\right), \quad z_{i+1}=\alpha_{i}-h\left(x_{i+1}^{*}\right) .
$$

The virtual control law $\alpha_{i}$ is designed as

$$
\alpha_{i}=-\frac{\ell_{i}}{\ell_{i+1}}\left(-c_{i} z_{i}-\dot{\alpha}_{i-1}-\frac{\ell_{i}}{\ell_{i-1}} z_{i-1}-\frac{\ell_{i+1}}{2 \ell_{i}}\right),
$$

where $c_{i}>0$ is designed constants. Invoking second equation of (22), (35), (36) and differentiating $z_{i}$ with respect to time yields

$$
\dot{z}_{i}=-c_{i} z_{i}+\frac{\ell_{i+1}}{\ell_{i}} z_{i+1}-\frac{\ell_{i}}{\ell_{i-1}} z_{i-1} .
$$

Consider the Lyapunov function as

$$
V_{i}=0.5 z_{i}^{2}
$$

Considering (37), the time derivative of $V_{i}$ is given by

$$
\dot{V}_{i}=-c_{i} z_{i}^{2}+\frac{\ell_{i+1}}{\ell_{i}} z_{i} z_{i+1}-\frac{\ell_{i}}{\ell_{i-1}} z_{i-1} z_{i} .
$$

Step $n$ Consider the last equation of system (22), Defining

$$
z_{n}=\alpha_{n-1}-h\left(x_{n}^{*}\right)
$$


Let $\bar{b}_{i}=\frac{b_{i} \ell_{i}}{2 \ell_{n}}, \bar{f}_{i}\left(x_{i}^{*}\right)=\frac{\mathrm{e}^{x_{i}^{*}-1}}{\mathrm{e}^{x_{i}^{*}}+1}, i=1,2, \cdots, n$. Then the $\dot{x}_{n}^{*}$ can be rewritten as

$$
\dot{x}_{n}^{*}=g\left(x_{n}^{*}\right)\left(\sum_{i}^{n} \bar{b}_{i} \bar{f}_{i}+\frac{1}{2 \ell_{n}}(\beta h(\nu)+f(\mathbf{0})+d(t))\right),
$$

where $\bar{f}_{i}$ is the abbreviation for $\bar{f}_{i}\left(x_{i}^{*}\right)$. Invoking (41) and differentiating $z_{n}$ yields

$$
\dot{z}_{n}=\bar{\beta} h(v)+\vartheta^{\mathrm{T}} \varphi
$$

where $\vartheta=\left[\bar{b}_{1}, \cdots, \bar{b}_{n}, f(\mathbf{0}) / 2 \ell_{n}, d(t) / 2 \ell_{n}, 1\right]^{\mathrm{T}}, \varphi=\left[\bar{f}_{1}, \cdots, \bar{f}_{n}, 1,1, \dot{\alpha}_{n-1}\right]^{\mathrm{T}}$ and $\bar{\beta}=\beta / 2 \ell_{n}$. The virtual control law $\alpha_{n}$ is designed as

$$
\left\{\begin{array}{l}
\alpha_{n}=N\left(\kappa_{0}\right) \varpi \\
N\left(\kappa_{0}\right)=\kappa_{0}^{2} \cos \left(\kappa_{0}\right), \\
\varpi=c_{n} z_{n}+\frac{\ell_{n}}{\ell_{n-1}} z_{n-1}+\hat{\vartheta}^{\mathrm{T}} \varphi+\mu^{2} z_{n} / 4 \chi
\end{array}\right.
$$

where $c_{n}>0$ and $\chi>0$ are designed constants and the other parameters are as follows.

$$
\begin{aligned}
& \mu \geq\left\|\tilde{\vartheta}_{\max }\right\|\|\varphi\|, \quad \tilde{\vartheta}_{\max }=\vartheta_{\max }-\vartheta_{\min }, \\
& \vartheta_{\max }=\left[\bar{b}_{1 \max }, \cdots, \bar{b}_{n \max }, \bar{d}_{0}, \bar{d}, 1\right]^{\mathrm{T}}, \\
& \vartheta_{\min }=\left[\bar{b}_{1 \min }, \cdots, \bar{b}_{n \min },-\bar{d}_{0},-\bar{d}, 1\right]^{\mathrm{T}}, \\
& \bar{b}_{i \max }=\frac{b_{i \max } \ell_{i}}{2 \ell_{n}}, \quad \bar{b}_{i \min }=\frac{b_{i \min } \ell_{i}}{2 \ell_{n}}, \quad i=1,2, \cdots, n, \\
& \bar{d}_{0}=\frac{d_{0}}{2 \ell_{n}}, \quad \bar{d}=\frac{d}{2 \ell_{n}} .
\end{aligned}
$$

The parameter updating laws are designed as

$$
\left\{\begin{array}{l}
\dot{\kappa}_{0}=\varpi z_{n}, \\
\dot{\hat{\vartheta}}=\operatorname{Proj}_{\hat{\vartheta}}\left(\Gamma \varphi z_{n}\right), \quad \hat{\vartheta}(0) \in \Omega_{\vartheta},
\end{array}\right.
$$

where $\Omega_{\vartheta}$ is the closed interval $\left[\vartheta_{\min }, \vartheta_{\max }\right]$, the $\tilde{\vartheta}=\vartheta-\hat{\vartheta}$ denotes the parameters projection errors. In this paper, the following adaptive algorithm with projection will be exploited.

$$
\operatorname{Proj}_{\hat{\vartheta}}(\cdot i)= \begin{cases}0, & \hat{\vartheta}=\vartheta_{\text {max }}, \cdot i>0, \\ 0, & \hat{\vartheta}=\vartheta_{\text {min }}, \cdot i<0, \\ \cdot i, & \text { other, }\end{cases}
$$

where $\Gamma$ is a diagonal matrix. It follows that the adaptive algorithm with projection assures that $\forall t: \hat{\vartheta}(t) \in \Omega_{\vartheta}$ is satisfied for all values of arguments ${ }^{[43]}$. Defining

$$
z_{n+1}=h(v)-\alpha_{n}
$$

Invoking (42)-(45), the $z_{n}$ can be expressed as

$$
\begin{aligned}
\dot{z}_{n} & =\bar{\beta} h(v)+\vartheta^{\mathrm{T}} \varphi \\
& =\bar{\beta} z_{n+1}+\bar{\beta} N\left(\kappa_{0}\right) \varpi+\vartheta^{\mathrm{T}} \varphi \\
& =-c_{n} z_{n}-\frac{\ell_{n}}{\ell_{n-1}} z_{n-1}+\tilde{\vartheta}^{\mathrm{T}} \varphi-\xi^{2} z_{n} / 4 \chi+\left(\bar{\beta} N\left(\kappa_{0}\right)+1\right) \dot{\kappa}_{0} / z_{n}+\bar{\beta} z_{n+1} .
\end{aligned}
$$


Consider the Lyapunov function as

$$
V_{n}=0.5 z_{n}^{2}
$$

Considering (47), the time derivative of $V_{n}$ is given by

$$
\dot{V}_{n}=-c_{n} z_{n}^{2}-\frac{\ell_{n}}{\ell_{n-1}} z_{n-1} z_{n}+\tilde{\vartheta}^{\mathrm{T}} \varphi-\mu^{2} z_{n}^{2} / 4 \chi+\left(\bar{\beta} N\left(\kappa_{0}\right)+1\right) \dot{\kappa}_{0}+\bar{\beta} z_{n} z_{n+1} .
$$

Using the inequality $a b \leq(1 / 4) a^{2}+b^{2}$ and substituting (43), (44) into (49) yields

$$
\begin{aligned}
\dot{V}_{n} \leq & -\left(c_{n}-1\right) z_{n}^{2}-\frac{\ell_{n}}{\ell_{n-1}} z_{n-1} z_{n}+\frac{\beta_{m}^{2} z_{n+1}^{2}}{4}+\left(\bar{\beta} N\left(\kappa_{0}\right)+1\right) \dot{\kappa}_{0} \\
& -\left(\left\|\tilde{\vartheta}_{\max }\right\|\|\varphi\|\left\|z_{n}\right\| / 2 \sqrt{\chi}-\sqrt{\chi}\right)^{2}+\chi \\
\leq & -\left(c_{n}-1\right) z_{n}^{2}-\frac{\ell_{n}}{\ell_{n-1}} z_{n-1} z_{n}+\frac{\beta_{m}^{2} z_{n+1}^{2}}{4}+\left(\bar{\beta} N\left(\kappa_{0}\right)+1\right) \dot{\kappa}_{0}+\chi,
\end{aligned}
$$

where $\beta_{m}=\max (|\bar{\beta}|)$.

Step $n+1$ The control input $\nu$ appears. The actual control law $\nu$ is designed as

$$
\left\{\begin{array}{l}
\dot{\nu}=-c \nu+\omega \\
\omega=N(\kappa)\left(c_{n+1} z_{n+1}-\eta c \nu-\dot{\alpha}_{n}\right), \\
N(\kappa)=\kappa^{2} \cos (\kappa)
\end{array}\right.
$$

The parameter updating law is designed as

$$
\dot{\kappa}=\left(c_{n+1} z_{n+1}-\eta c \nu-\dot{\alpha}_{n}\right) z_{n+1} .
$$

Invoking (51), (52), differentiating $z_{n+1}$ in (46) yields

$$
\begin{aligned}
\dot{z}_{n+1}=\frac{\partial h(\nu)}{\partial \nu} \dot{\nu}-\dot{\alpha}_{n} & =\eta(-c \nu+\omega)-\dot{\alpha}_{n} \\
& =-c_{n+1} z_{n+1}+(\eta N(\kappa)+1) \dot{\kappa} / z_{n+1} .
\end{aligned}
$$

Consider the Lyapunov function as

$$
V_{n+1}=0.5 z_{n+1}^{2} .
$$

Considering (53), the time derivative of $V_{n+1}$ is given by

$$
\dot{V}_{n+1}=z_{n+1} \dot{z}_{n+1}=-c_{n+1} z_{n+1}^{2}+(\eta N(\kappa)+1) \dot{\kappa} \leq-\lambda_{0} V_{n+1}+(\eta N(\kappa)+1) \dot{\kappa},
$$

where $\lambda_{0} \leq 2 c_{n+1}$. Let $\delta_{0}=V_{n+1}(0)$ and multiplying both sides in the above inequality by $\mathrm{e}^{\lambda_{0} t}$ and integrating over $[0, t]$, it can be obtained that

$$
V_{n+1}(t) \leq \delta_{0}+\mathrm{e}^{-\lambda_{0} t} \int_{0}^{t}(\eta N(\kappa)+1) \dot{\kappa} \mathrm{e}^{\lambda_{0} \iota} \mathrm{d} \iota .
$$

According to the Lemmas 1 and 2, we can obtain that $V_{n+1}$ and $z_{n+1}$ are bounded, and assume that $\left|z_{n+1}\right| \leq \bar{z}_{n+1}$, where $\bar{z}_{n+1}=\max \left(\left|z_{n+1}\right|\right)$. Since that the signals in step $n+1$ are bounded, consider the convergent property of other signals in steps 1 to $n$, choose the Lyapunov function as

$$
V=\sum_{i=1}^{n} V_{i}
$$


Considering (28), (34), (39), (50) and differentiating $V$ yields

$$
\begin{aligned}
\dot{V} & \leq \sum_{i=1}^{n-1}-c_{i} z_{i}^{2}-\left(c_{n}-1\right) z_{n}^{2}+\frac{\beta_{m}^{2} z_{n+1}^{2}}{4}+\left(\beta N\left(\kappa_{0}\right)+1\right) \dot{\kappa}_{0}+\chi \\
& \leq-\lambda V+\chi+\beta_{m}^{2} \bar{z}_{n+1}^{2} / 4+\left(\bar{\beta} N\left(\kappa_{0}\right)+1\right) \dot{\kappa}_{0},
\end{aligned}
$$

where $\lambda=\min \left(2 c_{i}, 2 c_{n}-2\right), i=1,2, \cdots, n-1$. Let $\delta=V(0)+\chi / \lambda+\beta_{m}^{2} \bar{z}_{n+1}^{2} / 4 \lambda$, multiplying both sides in the above inequality by $\mathrm{e}^{\lambda t}$, and integrating over $[0, t]$, it can be obtained that

$$
V(t) \leq \delta+\mathrm{e}^{-\lambda t} \int_{0}^{t}\left(\bar{\beta} N\left(\kappa_{0}\right)+1\right) \dot{\kappa}_{0} \mathrm{e}^{\lambda \tau} \mathrm{d} \tau
$$

Thus, according to the Lemma 1 and 2, all signals of the closed-loop system are bounded, that is, the tracking error of the system (22) is bounded. According to characteristic of nonlinear mapping, the following conclusions can be obtained.

(i) Considering inequality (59) and Lemmas $1-2$, we know that $x_{i}^{*}, z_{i}(i=1,2, \cdots, n), y_{r}^{*}, \kappa_{0}, \kappa$ and $\hat{\vartheta}$ are bounded. Thus according to Equations (9), (10) and Lemma 3, it obtained that $x_{i}$ and $y_{r}$ are bounded and $x_{i}$ always remains in the set $\left|x_{i}\right| \leq \ell_{i}$.

(ii) According to NMT, PPB and Lemma 3, the tracking error $e(t)=y-y_{r}$ is preserved within a specified PPB at all times.

According to above analysis, the following theorem is proposed.

Theorem 1 Considering the nonaffine system (1) with parameter uncertainty, state constraints, input constraint and unknown external disturbance, the parameter adaptive laws are designed as (44) and (52), and the virtual control laws are designed as (25), (30), (36) and (43). Under the adaptive control scheme (51), all signals of the closed-loop system are bounded. Furthermore, the transient performance of the system in the sense that $e(t)=y-y_{r}$ is preserved within a specified $P P B$ at all times, i.e., $-\varsigma \rho(t)<e(t)<\bar{\varsigma} \rho(t)$ with $t \geq 0$, is guaranteed.

Proof It is apparent that Theorem 1 can be easily proved via the inequality (59) when only the appropriate design parameter $c, c_{i}(i=1,2, \cdots, n), \chi$ and $\Gamma$ are chosen.

Remark 3 In order to solve the "explosion of complexity" problem in backstepping technique, we can employ the differentiator ${ }^{[21]}$ as follows

$$
\left\{\begin{array}{l}
\dot{v}_{1}=v_{2}, \\
\dot{v}_{2}=-\tau^{2} \operatorname{sgn}\left(v_{1}-\alpha_{i}\right)\left|v_{1}-\alpha_{i}\right|^{\varsigma}-\tau v_{2},
\end{array}\right.
$$

where $\tau>0,0<\varsigma<1$, and $\alpha_{i}(i=1,2, \cdots, n)$ is the virtual control. Then $v_{2}$ can indicate $\dot{\alpha}_{i}$ commendably.

\section{Simulation Result and Analysis}

In this section, the feasibility of the proposed scheme and the control performances are illustrated by the following example.

$$
\left\{\begin{array}{l}
\dot{x}_{1}=x_{2} \\
\dot{x}_{2}=f\left(x_{1}, x_{2}, u\right)+d_{m}(t), \\
y=x_{1}
\end{array}\right.
$$


where uncertain nonlinear function $f(\cdot)$ is expressed as

$$
f(\cdot)= \begin{cases}-0.1 x_{1}-0.25 x_{2}-x_{1}^{3}+u^{3}+\left(2+\cos x_{2}\right) u, & t \leq 15 \\ -(0.2 \sin (10 t)) x_{1}-(0.2+0.2 \cos (5 t)) x_{2} & \\ -x_{1}^{3}+(\omega u)^{3}+\left(2+\cos x_{2}\right) \omega u+\zeta, & 15<t\end{cases}
$$

where $\omega$ is the health indicator reflecting the effectiveness of the actuator and $\zeta=\sin \omega u$ is the uncertain partition of the control that is completely out of control, i.e., system (61) exists actuator failure with the time increasing, in simulation, the $\omega$ is chosen as 0.85 . The $d_{m}(t)$ is expressed as

$$
d_{m}(t)= \begin{cases}0, & t \leq 10 \\ \sin (0.1 t), & 10<t \leq 20 \\ \sin t+0.5 \cos (0.5 t), & 20<t\end{cases}
$$

The constrained state and input are $\left|x_{1}\right| \leq 1$, and $u_{M}=5$. According the mean value theorem, the $b_{1}(t), b_{2}(t)$ and $\beta(t)$ can be expressed as follows

$$
\begin{aligned}
& b_{1}(t)=\frac{\partial f}{\partial x_{1}}= \begin{cases}-0.1-3 x_{1}^{2}, & t \leq 15, \\
-0.2 \sin (10 t)-3 x_{1}^{2}, & 15<t, \\
-0.25-u \sin x_{2}, & t \leq 15, \\
-(0.2+0.2 \cos (5 t))-\omega u \sin x_{2}, & 15<t,\end{cases} \\
& b_{2}(t)=\frac{\partial f}{\partial x_{2}}= \\
& \beta(t)=\frac{\partial f}{\partial u}= \begin{cases}3 u^{2}+2+\cos x_{2}, & t \leq 15, \\
3 \omega^{3} u^{2}+\left(2+\cos x_{2}\right) \omega+\omega \cos (\omega u), & 15<t .\end{cases}
\end{aligned}
$$

Since $\left|x_{1}\right| \leq 1, u_{M}=5,|\sin (\cdot)| \leq 1$ and $|\cos (\cdot)| \leq 1$, we can obtain that the $b_{1}(t), b_{2}(t)$ and $\beta(t)$ satisfy

$$
\left\{\begin{array} { l } 
{ b _ { 1 } ( t ) \in [ - 3 . 1 , 2 . 9 ] , t \leq 1 5 , } \\
{ b _ { 1 } ( t ) \in [ - 3 . 2 , 3 . 2 ] , 1 5 < t , }
\end{array} \quad \left\{\begin{array} { l } 
{ b _ { 2 } ( t ) \in [ - 5 . 2 5 , 4 . 7 5 ] , t \leq 1 5 , } \\
{ b _ { 2 } ( t ) \in [ - 4 . 6 2 , 4 . 2 5 ] , 1 5 < t , }
\end{array} \quad \left\{\begin{array}{l}
\beta(t) \in[76,78], \quad t \leq 15, \\
\beta(t) \in[46.1,49.5], 15<t .
\end{array}\right.\right.\right.
$$

It is easy to know that the the $b_{1}(t), b_{2}(t)$ and $\beta(t)$ must be restricted in the following intervals in the whole time of system running.

$$
b_{1}(t) \in[-3.2,3.2], \quad b_{2}(t) \in[-5.25,4.75], \quad \beta(t) \in[76,78] .
$$

Since $d=\max (|\beta|) u_{M}(1-\tanh (1))+d_{m}$, invoking (63), we can obtain $|d(t)| \leq 94.476$. Based on the constraint intervals of $b_{1}(t), b_{2}(t)$ and $d(t)$, we can design controller for system (61) by employing (25), (30), (36), (43), (44), (51) and (52).

All the design parameters for system (61) in the simulation study are chosen as $c_{1}=2$, $c_{2}=0.5, c_{3}=10, c=0.15, \chi=500$ and $\Gamma=\operatorname{diag}(0.05)_{5 \times 5}$. The initial state conditions are chosen as $x(0)=[0.5,0]^{\mathrm{T}}$, The reference trajectory is taken as $y_{r}=0.5 \sin (0.5 t)+0.5 \sin (t)$.

It is easy to obtain $y_{r}(0)=0$. So the $\rho_{0}$ can be designed as $\rho_{0} \geq \ln ((1+0.5) /(1-0.5))+0.5=$ 1.5986, then the decreasing smooth function for the PPB is designed as $\rho(t)=1.1 \mathrm{e}^{-t}+0.5$.

The tracking results, the control input, the tracking error and adaptive parameters are shown in Figures 1 to 5 under the proposed adaptive control scheme considering PPB. From 
Figure 1, we observe that the tracking performance is satisfactory for the system taking into account the unknown external disturbance, the state constraints and the input saturation. From Figure 2, for the time-varying reference signals, the tracking errors remain within the prescribed tracking error bounds. The control input is shown in Figure 3 which is bounded and convergent. From Figures 4 and 5, it is obvious that all the parameters are bounded.

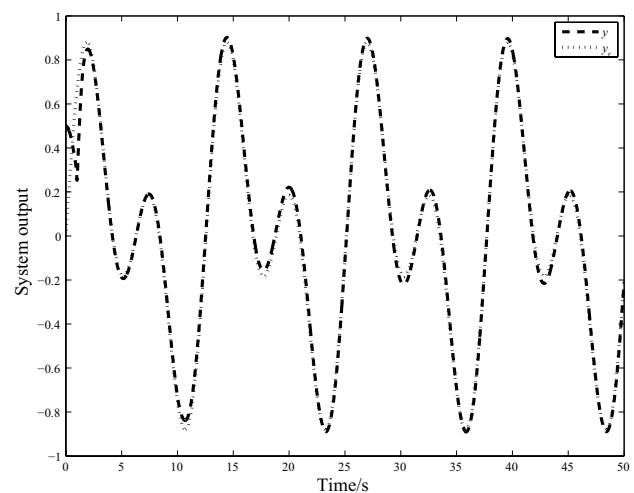

Figure 1 System output $y$ and reference signal $y_{r}$

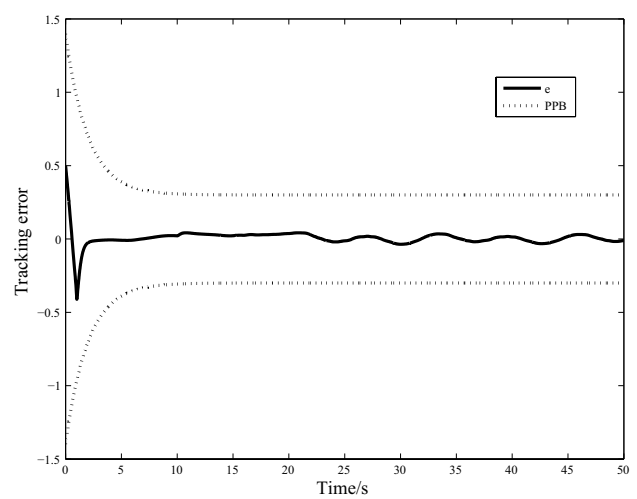

Figure 2 System tracking error

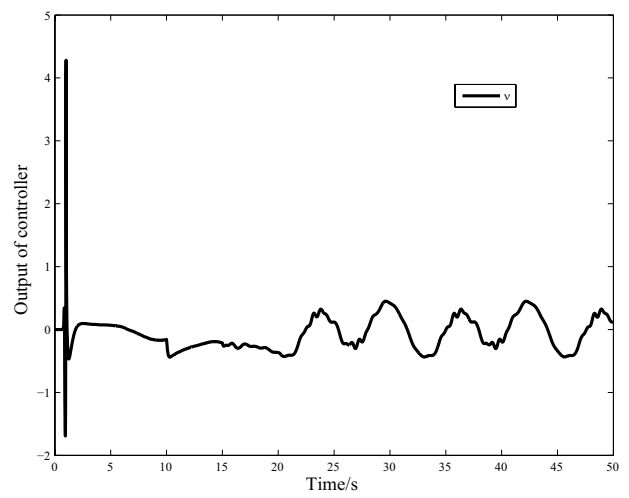

Figure 3 Output of controller

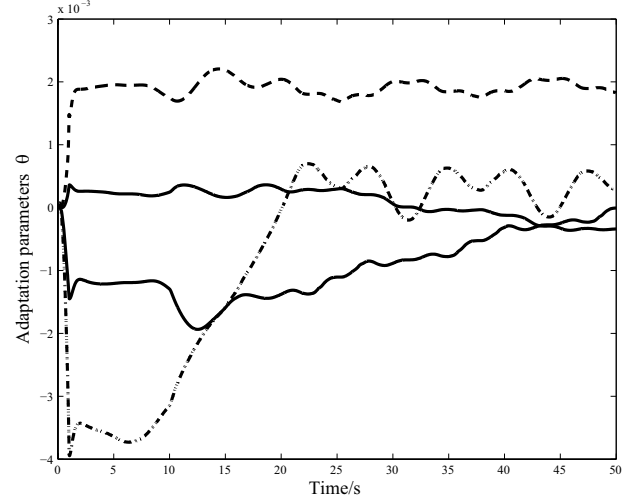

Figure 4 Adaptation of parameters $\kappa_{0}$ and $\kappa$

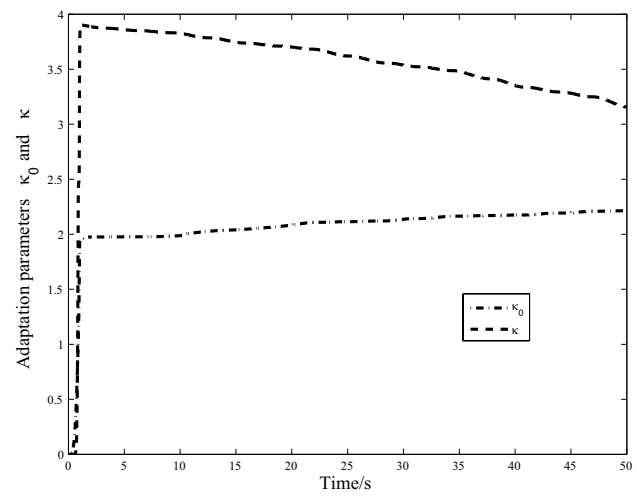

Figure 5 Adaptation of parameters $\hat{\vartheta}$ 
From the above simulation results, we conclude that the proposed adaptive control scheme is robust, adaptable, fault-tolerant and universal for the nonaffine nonlinear system with state constraints, input constraint, uncertain time-varying parameters and unknown external disturbance.

\section{Conclusion}

In this paper, an effective adaptive control scheme has been proposed for non-affine system in the presence of state constraints, input constraint, uncertain parameters and unknown external disturbance. Considering the state and input constraints, the NMT, hyperbolic tangent function and Nussbaum function are employed. The prescribed performance control method improves the performance of the system. The designed scheme can not only deal with the constraints but also the possible "controller singularity" problem causing by unknown control direction. Our future research efforts will focus on that the bound of external disturbance are unknown, the control input contains nonsymmetric nonlinearities of saturation as well as dead zone and the pure feedback non-affine system.

\section{References}

[1] Krstic M, Kanellakopoulos I, Kokotovic P V. Nonlinear and Adaptive Control Design. Wiley, New York, 1995.

[2] Boskvoic J D, Chen L J, Mehra R K. Adaptive control design for nonaffine models arising in flight control. Journal of Guidance, Control and Dynamics, 2004, 27(2): 209-217.

[3] Shiriaev A S, Ludvigsen H, Egeland O, et al. Swinging up of non-affine in control pendulum. Proceedings of American Control Conference, San Diego, California, USA, 1999: 4039-4044.

[4] Tang H M, Li C W. $H_{\infty}$ robust control of AC/DC systems with non-affine nonlinear model. Acta Automatica sinica, 2007, 33(7): 709-713.

[5] Karimi B, Menhaj M B. Non-affine nonlinear adaptive control of decentralized large-scale systems using neural networks. Information Sciences, 2010, 180(17): 3335-3347.

[6] Song Q, Song Y D. PI-like fault-tolerant control of nonaffine systems with actuator failures. Acta Automatica Sinica, 2012, 38(6): 1033-1040.

[7] Shiriaev A S, Fradkov A L. Stabilization of invariant sets for nonlinear non-affine systems. Automatica, 2000, 36(11): 1709-1715.

[8] Arefi M M, Zarei J, Karimi H R. Adaptive output feedback neural network control of uncertain non-affine systems with unknown control direction. Journal of the Franklin Institute, 2014(351): 4302-4316.

[9] Zhou W D, Liao C Y. Indirect adaptive fuzzy output-feedback controller for a SISO nonaffine system with unknown control direction. Control Theory \& Applications, 2013, 30(9): 1131-1137.

[10] Park J Y, Park G T. Robust adaptive fuzzy controller for nonaffine nonlinear systems with dynamic rule activation. Int. J. Robust Nonlinear Control, 2003, 13(2): 117-139.

[11] Labiod S, Guerra T M. Indirect adaptive fuzzy control for a class of non-affine nonlinear systems with unknown control directions. International Journal of Control, Automation, and Systems, 2010, 8(4): 903907.

[12] Zhang Q, Wu Q X, Jiang C S, et al. Robust control for nonaffine nonlinear systems based on Backstepping. Control and Decision, 2014, 29(1): 19-26.

[13] Chen L S, Wang Q. Adaptive robust control for a class of uncertain non-affine nonlinear system. Control Theory \& Applications, 2015, 32(2): 256-261.

[14] Shen Q K, Shi P, Zhang T P, et al. Novel neural control for a class of cuncertain pure-feedback systems. IEEE Transactions on Neural Network and Learning Systems, 2014, 25(4): 718-727.

[15] Ge S S, Wang C. Adaptive NN control of uncertain nonlinear pure-feedback systems. Automatica, 2002(38): 671-682.

[16] Sun G, Wang D, Li X Q, et al. A DSC approach to adaptive neural network tracking controlfor purefeedback nonlinear systems. Applied Mathematics and Computation, 2013, 219: 6224-6235. 
[17] Labiod S, Guerra T M. Adaptive fuzzy control of a class of SISO nonaffine nonlinear systems. Fuzzy Sets Syst., 2007, 158(10): 1126-1137.

[18] Park J H, Park G T, et al. Direct adaptive self-structuring fuzzy controller for nonaffme nonlinear system. Fuzzy Sets and Systems, 2005, 153(3): 429-445.

[19] Wen J, Jiang C S. Adaptive fuzzy control for a class of chaotic systems with nonaffine inputs. Commun. Nonlinear Sci. Numer. Simulat., 2011, 16: 475-492.

[20] Cheng $\mathrm{C} \mathrm{H}, \mathrm{Wu} \mathrm{J} \mathrm{H}, \mathrm{Hu} \mathrm{Y} \mathrm{A}$, et al. Adaptive control of non-affine nonlinear system with saturation constraint. Control Theory \& Applications, 2014, 31(8): 1000-1008.

[21] Cheng C H, Hu Y A, Wu J H. Auto disturbance controller of non-affine nonlinear pure feedback systems. Acta Automatica Sinica, 2014, 40(7): 1528-1535.

[22] Song Q, Song Y D. PI-like fault-tolerant control of nonaffine systems with actuator failures. Acta Automatica Sinica, 2012, 38(6): 1033-1040.

[23] Song R Z, Zhang H G, Luo Y H, et al. Optimal control laws for time-delay systems with saturating actuators based on heuristic dynamic programming. Neurocomputing, 2010, 73(16-18): 3020-3027.

[24] Luo Y H, Zhang H G. Approximate optima lcontrol for a class of nonlinear discrete-time systems with saturating actuators, Prog. Nat. Sci., 2008, 18(8): 1023-1029.

[25] Mayne D, Rawlings J, Rao C, et al. Constrained model predictive control: Stability and optimality. Automatica, 2000, 36(6): 789-814.

[26] Patwardhan A, Rawlinfs J, Edagr T. Nonlinear model predictive control. Chemical Engineering Communications, 1990, 87(1): 123-141.

[27] Do H M, Basar T, Choi J Y. An anti-windup design for single input adaptive control systems in strict feedback form. Proc. Amer. Control Conf., 2004: 2551-2556.

[28] Chen M, Ge S S, Ren B B. Adaptive tracking control of uncertain MIMO nonlinear systems with input constraints. Automatica, 2011, 47(3): 452-465.

[29] Li Y M, Li T S. Adaptive fuzzy output-feedback control for output constrained nonlinear systems in the presence of input saturation. Fuzzy Sets and Systems, 2014, 248: 138-155.

[30] Wen C Y, Zhou J, Liu Z T, et al. Robust adaptive of uncertain nonlinear systems in the presence of input saturation and external disturbance. IEEE Trans. Autom. Control, 2011, 56(7): 1672-1678.

[31] Li Y M, Tong S C, Li T S. Direct adaptive fuzzy backstepping control of uncertain nonlinear systems in the presence of input saturation. Neural Comput. Appl., 2013, 23(5): 1207-1216.

[32] Ngo K B, Mahony R, Jiang Z P. Integrator backstepping using barrier functions for systems with multiple state constraints. Proc. 44th IEEE Conf. Decision and Control, 2005: 8306-8312.

[33] Liu Y J, Li D J, Tong S C. Adaptive output feedback control for a class of nonlinear systems with full-state constraints. International Journal of Control, 2014, 87(2): 281-290.

[34] Zhang S J, Qiu X W, Liu C S. Neural adaptive compensation control for a class of MIMO uncertain nonlinear systems with actuator failures. Circuits Syst. Signal Process, 2014, 33: 1971-1984.

[35] Bechlioulis C P, Rovithaki G A. Prescribed performance adaptive control for multi-input multi-output affine in the control nonlinear systems. IEEE Trans. on Auto. Contr., 2010, 55(5): 1220-1226.

[36] Bechlioulis C P, Rovithaki G A. Robust adaptive control of feedback linearizable MIMO nonlinear systems with prescribed performance. IEEE Trans Auto. Contr., 2008, 53: 2090-2099.

[37] Zhang Y, Wen C Y, Soh Y C. Robust decentralized adaptive stabilization of interconnected systems with guaranteed transient performance. Automatica, 2000, 36: 907-915.

[38] Li Y H, Sheng Q, Zhang X Y. Roubust and adaptive backstepping control for nonlinear systems using RBF neural network. IEEE Trans. on Neural Networks, 2004, 15(3): 693-701.

[39] Zhang Y, Peng P Y, Jiang Z P. Stable neural controller design for unknown nonlinear systems using backstepping. IEEE Transactions on Neural Networks, 2000, 11(6): 1347-1360.

[40] Han J Q. Active Disturbance Rejection Control Technique — The Technique for Estimating and Compensating the Uncertainties. Beijing: National Defense Industry Press, 2009: 56-66.

[41] Boulkrounea A, Tadjine M, Saad M M, et al. Fuzzy adaptive controller for MIMO nonlinear systems with known and unknown control direction. Fuzzy Sets and Systems, 2010(161): 797-820.

[42] Swaroop D, Hedrick J K, Yip P P. Dynamic surface control for a class of nonlinear systems. IEEE Transactions on Automatic Control, 2000, 45(10): 1893-1899. 